\title{
171 NEUROPATHY PHENOTYPE IN HEREDITARY TRANSTHYRETIN AMYLOIDOSIS
}

Aisling Carr, Ana Pelayo, AD Wechalekar, CJ Whelan, JD Gilmore, PN Hawkins, MM Reilly. UCL; NAC

\subsection{6/jnnp-2014-309236.171}

Background Hereditary transthyretin amyloidosis (ATTR) is associated with progressive peripheral neuropathy, cardiac, gastrointestinal and autonomic failure due to dominantly inherited transthyretin mutations causing amyloid deposition. The neuropathy phenotype is less well described than cardiac manifestations.

Methods Detailed clinical neurological and electrophysiological data were collected on all patients. Follow-up data was available on a subset.

Results Thirtyfour patients (mean age $=62$ years; $70.6 \%$ male) were assessed atleast once; 19 (55.8\%) had serial examinations, mean follow-up: 1.9 years. The genetic breakdown was $38.2 \%$ T60A, 14.7\% V30M, 8.8\% I107F, 5.9\% V122I, 32.4\% individual mutations; $76.5 \%$ UK and/or Irish ancestry. 44.1\% were treated (Diflunasil or liver transplant). $26.5 \%$ presented with neuropathy; $70.6 \%$ had neuropathy on follow-up. A lengthdependant, axonal, sensorimotor neuropathy was typical; $6.7 \%$ had patchy onset, $10 \%$ had demyelinating features. Mean MRC score at presentation was 62.5 with a mean reduction of 2.7 points/year and a trend to slower deterioration in the treated group (2.4 versus 3.0 points/year; $\mathrm{p}=0.77$ ).

Conclusion The detection of measurable annual changes in MRC score is encouraging but monitoring of sensory deficits should also be quantifiable. This small but representative study mirrors difficulties observed in recent treatment trials regarding sensitivity of current outcome measures. 